\title{
Inflammatory Microenvironment-Mediated E-Cadherin Decrease Induces Migration in LNCaPs ${ }^{\dagger}$
}

\author{
Iroda Saydullaeva ${ }^{1}$, Bilge Debeleç Bütüner ${ }^{2}$ and Kemal Sami Korkmaz 1,* \\ 1 Department of Bioengineering, Ege University, 35040 Bornova, Izmir, Turkey; iroda_55@hotmail.com \\ 2 Department of Pharmaceutical Biotechnology, Faculty of Pharmacy, Ege University, 35040 Bornova, Izmir, \\ Turkey; bilged79@hotmail.com \\ * Correspondence: ks_korkmaz@yahoo.com \\ + Presented at the 2nd International Cell Death Research Congress, Izmir, Turkey, 1-4 November 2018.
}

Published: 6 December 2018

\begin{abstract}
Infection and chronic inflammation contribute to about 1 in 4 of all cancer cases. Emerging evidence suggests that the host factors in the tumor microenvironment may interact with underlying inflammatory prostate cancer cells to make them aggressive. In this study, we hypothesized that soluble factors secreted by immune cells activated by inflammation can induce EMT in prostate cancer and thus promote metastasis. We identify that macrophage-secreted cytokines including TNF $\alpha$ act as mediators for potentiating LNCaP cell proliferation in migration assay. Hence, our data indicate a mechanistic insight of how inflammation may contribute to development of prostatic disease at an early stage through increasing cell proliferation and metastasis of LNCaP cells.
\end{abstract}

Keywords: inflammation; TNF $\alpha$; prostate cancer

\section{Introduction}

Prostate cancer is the second leading cause of cancer-associated deaths among men in the western world [1]. As with most cancers, prostate cancer progression is characterized by several alterations at the genome level including both germline and somatic mutations [2]. Prostate disorders are associated with an increase of inflammation [3] and elevated cell proliferation of prostate cells [4]. A sustained inflammatory cell environment and uncontrolled cell proliferation, both of which can lead to tumorigenesis. Many mechanisms have been identified in this process that can be rationalized into targeted therapy. Among them, epithelial-to-mesenchymal transition (EMT) is originally characterized as a critical step for cell trans-differentiation during embryo development and now recognized in promoting cancer cells invasiveness because of high mobility and migratory abilities of mesenchymal cells once converted from carcinoma cells [5]. EMT occurs by breakdown of cell-to-cell or cell-to-extracellular matrix (ECM) adherence at the polarized epithelium lining. E-cadherin is a major component of epithelial adherence junction and acts as the master gatekeeper of EMT. Loss of E-cadherin, considered to be the key step to initiate EMT, leads to collapse of intercellular mechanical communication [6].

\section{Materials and Methods}

\subsection{Macrophage Differentiation and Conditioned Media (CM) Collection}

The U937 monocyte cell line was cultured in RPMI 1640 medium including 10\% FBS (fetal bovine serum) at $37{ }^{\circ} \mathrm{C}$ in the presence of $5 \% \mathrm{CO}_{2}$. To achieve macrophage differentiation and cytokine production, cells $\left(8 \times 10^{5}\right)$ were seeded into $75 \mathrm{~cm}^{2}$ culture flasks for $2 \mathrm{~h}$ prior to the treatments. Next, PMA was added at concentrations of $16 \mathrm{nM}$ for $16 \mathrm{~h}$, and the adherent clusters 
were followed. The cells were washed, and $20 \mathrm{~mL}$ of fresh medium was added. After allowing the cells to rest for $2 \mathrm{~h}$, lipopolysaccharide (LPS) was added at a final concentration of $10 \mathrm{ng} / \mathrm{mL}$, and the cells were incubated for $24 \mathrm{~h}$. Finally, the supernatant conditioned medium (CM) was collected and filtered using a 0.8 and $0.22 \mathrm{~mm}$ filters for further use.

\subsection{Measurement of Cytokines in the $C M$}

Before feeding the LNCaP cells with the collected CM, TNF $\alpha$ (Invitrogen, Carlsbad, CA, USA), levels were examined using an ELISA according to the manufacturer's instructions.

\subsection{Cell Culture and Treatments}

LNCaP cells were obtained from the American Type Culture Collection (ATCC, Manassas, VA, USA) and were propagated as recommended using RPMI 1640 medium supplemented with $10 \%$ FBS, L-glutamine $(2 \mathrm{mM})$, penicillin $(100 \mathrm{U} / \mathrm{mL})$ and streptomycin $(100 \mathrm{mg} / \mathrm{mL})$. All experiments were carried out using cells grown in a humidified incubator containing $5 \% \mathrm{CO}_{2}$ at $37^{\circ} \mathrm{C}$. The acute inflammation treatments were performed with CM $(500 \mathrm{pg} . / \mathrm{mL} \mathrm{TNF} \alpha)$ at appropriate period of $12 \mathrm{~h}$. TNF $\alpha$ concentrations were adjusted by diluting the CM using RPMI 1640 medium.

\subsection{Cell Migration Assay}

The cell migration assay was carried out using Transwell inserts placed in 6-well plates, which had a pore size of $8.0 \mu \mathrm{m}$ (Corning, NY, USA). To determine migration in LNCaP cells, the cells were seeded in $10 \mathrm{~cm}$ plates and allowed to reach $70 \%-80 \%$ confluence, followed by incubation with or without CM, including $500 \mathrm{pg}$./mL TNF $\alpha$ for $12 \mathrm{~h}$. After incubation approximately $3 \times 10^{5}$ cells in 1.5 mL RPMI-1640 culture medium without FBS were replaced in the upper compartment of 6-well plates, and the lower compartment was filled with 2 mL RPMI-1640 supplemented with $10 \%$ FBS. LNCaP cells were incubated for $62 \mathrm{~h}$ at $37^{\circ} \mathrm{C}$ in $5 \% \mathrm{CO}_{2}$. After transwell inserts were removed and cells migrated to the lower compartment have been grown for next step analyses.

\subsection{Sub-Cellular Fractionation}

Briefly, in order to obtain membrane, cytoplasmic and nuclear fractions, migrated cells $\left(2 \times 10^{7}\right)$ were washed with PBS and pelleted for $5 \mathrm{~min}$ at $300 \mathrm{~g}$. The cell pellet resuspended in buffer. Then the subcellular fractionation was performed using differential centrifugation, which was described in previous publications [7]. All incubations and centrifugations were carried out at $4{ }^{\circ} \mathrm{C}$, buffers were supplemented with $1 \times$ protease inhibitor cocktail (Roche GmbH, Germany) and $1 \mathrm{mM}$ DTT directly before use. $50 \mathrm{mg}$ of each fraction was subjected to SDS-PAGE.

\subsection{Western Blot Analysis}

Protein concentrations were determined using BCA assay (Sigma, UK). SDS-PAGE and western blots were performed under standard conditions using $50 \mathrm{mg}$ of protein lysate per lane; proteins were separated on a 10-20\% gel and transferred to PVDF membrane (Amersham, UK) using a wet transfer blotter. The PVDF membrane was blocked with 5\% dry milk in TBS-T (Tris-Borate-Saline solution containing $0.1 \%$ Tween 20 ). Primary and secondary antibody incubations were performed using TBS-T containing $0.5 \%$ dry milk or $5 \%$ BSA at RT for $1 \mathrm{~h}$ or at $4{ }^{\circ} \mathrm{C}$ for o/n. Membranes were developed using ECL plus reagent (Amersham, UK) for $5 \mathrm{~min}$ and were photographed using Kodak X-ray films in a dark room.

\section{Results}

\subsection{Induces LNCaP Cell Migration}

In this study, we aimed to characterize the EMT as well as migration-related molecular changes induced by inflammatory microenvironment. To observe the role of inflammatory microenvironment in prostate cells migration, conditioned media including $500 \mathrm{pg} / \mathrm{mL} \mathrm{TNF} \alpha$ was 
added onto the LNCaP cells for $12 \mathrm{~h}$. After treatment, these cells were accommodated to transwell inserts to set up migration assay (Figure 1). After 62 hours of incubation, we measured the migration capacity of the $\mathrm{CM}$ treated cells and found that it increased. These findings suggest that the inflammatory cytokines may be involved in LNCaP cells migration.

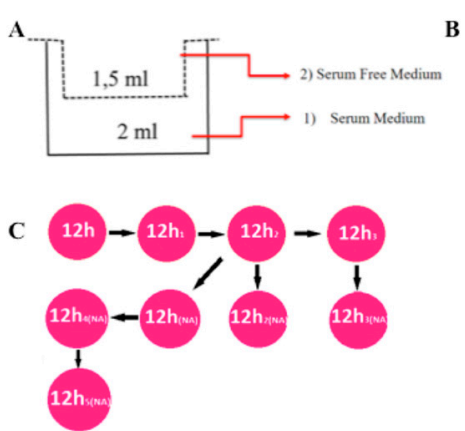

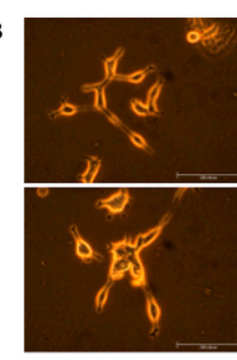

Control

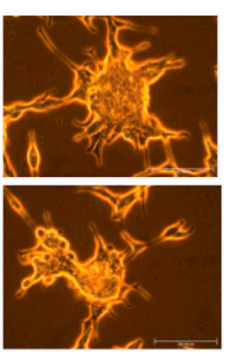

CM 12h

Figure 1. CM treatment induces migration in LNCaP cells. (A) The diagram for demonstrating migration assay with Transwell inserts. (B) CM treated and control cells incubated and cultured after migration for a month until enough number of cells were obtained for further experiments. Number of migrated cells after CM treatment was more than control (data not shown) group, but in the first month, possibly because of CM-induced-differentiation, proliferation rate of treated cells was lower then control cells. After this period CM treated cells have become more aggressive. (C) In the period of incubation, some cells observed to carry characteristics of suspension cell and these cells were separated and grown clonally. Each time when we passage these nonadhesive cells, some of them have become adhesive and others have maintained nonadhesive (NA) properties. Clones were named according to their adhesion properties.

\subsection{Decreased E-Cadherin Expression in Inflammation-Induced Migration of Prostate Cancer Cells}

Cells obtained after Transwell migration assay were undertaken to validate the protein levels of E-cadherin as an EMT marker and p-Histone 3-Ser10 as a proliferation marker. Membrane, cytoplasmic and nuclear proteins were fractionated and subcellular fractionation coupled western blotting revealed that E-cadherin protein levels decreased in CM treated and migrated cells. Concurrently, $\mathrm{p}$-Histone 3-Ser10 levels increased in the same group of cells. $\beta$-actin expression was used as loading control (Figure 2). Taken together, these results indicate that loss of E-cadherin expression contributes to epithelial-mesenchymal transition and consequently to migration of LNCaP cells.

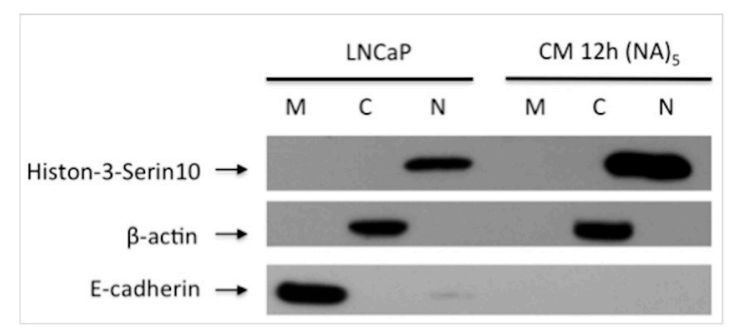

Figure 2. Alteration of Expression E-cadherin and phospho-Histone $3^{\text {S10 }}$ in the Inflammatory Microenvironment. In the migrated cells, E-cadherin expression was decreased, meanwhile Histone 3-Ser10 was increased then LNCaP cells. M: Membrane, C: Cytoplasmic, N: Nuclear fraction after subcellular protein isolation. CM $12 \mathrm{~h}(\mathrm{NA}) 5$ : 5th generation cells obtained after migration assay and changed their properties as a nonadhesive. 


\section{Discussion}

The elevated inflammation during the development of prostate cancer is etiologically recognized in many. Since the major contributing factors need to be assessed, some studies reported that the macrophage differentiation-mediated cytokine release is an important factor for this phenomenon. It has been previously shown that inflammatory microenvironment increases LNCaP cell migration and deregulation of membrane localized $\beta$-catenin function may promote tumorigenesis by altering gene transcription, increasing cell migration and abrogating cell polarity [7]. Therefore, in our studies, we hypothesized that the inflammation mediated EMT could be one of the promoting factors of prostate cancer development. Then, we assessed the migration abilities of the cells upon conditioned media treatments involving predetermined concentrations of the major pro-inflammatory cytokines such as TNF $\alpha$. We observed that the membrane-bound E-cadherin levels significantly decreased indicating the induced EMT after inflammatory conditions where the migrated cells exhibited higher Histone H3 Ser10 phosphorylation suggesting the increased mitotic rate of migrated cells.

Acknowledgments: This work was supported by The Scientific and Technological Research Council of Turkey (TÜBITAK, Project no: 117S289).

\section{References}

1. Siegel, R.; Ma, J.; Zou, Z.; Jemal, A. Cancer statistics, 2014. CA Cancer J. Clin. 2014, 64, 9-29.

2. Boyd, L.K.; Mao, X.; Lu, Y.J. The complexity of prostate cancer: Genomic alterations and heterogeneity. Nat. Rev. Urol. 2012, 9, 652-664.

3. Ellem, S.J.; Wang, H.; Poutanen, M.; Risbridger, G.P. Increased endogenous estrogen synthesis leads to the sequential induction of prostatic inflammation (prostatitis) and prostatic pre-malignancy. Am. J. Pathol. 2009, 175, 1187-1199.

4. Kim, J.H.; Kim, S.S.; Han, I.H.; Sim, S.; Ahn, M.H.; Ryu, J.S. Proliferation of Prostate Stromal Cell Induced by Benign Prostatic Hyperplasia Epithelial Cell Stimulated with Trichomonas vaginalis via Crosstalk with Mast Cell. Prostate 2016, 76, 1431-1444.

5. Lo, U.G.; Lee, C.F.; Lee, M.S.; Hsieh, J.T. Review the Role and Mechanism of Epithelial-to-Mesenchymal Transition in Prostate Cancer Progression. Int. J. Mol. Sci. 2017, 18, 2079.

6. Grant, C.M.; Kyprianou, N. Epithelial-mesenchymal transition (EMT) in prostate growth and tumor progression. Transl. Androl. Urol. 2013, 2, 202-211.

7. Debelec-Butuner, B.; Alapinar, C.; Ertunc, N.; Gonen-Korkmaz, C.; Yörükoğlu, K.; Korkmaz, K.S. TNFa-Mediated Loss of b-Catenin/E-Cadherin Association and Subsequent Increase in Cell Migration Is Partially Restored by NKX3.1 Expression in Prostate Cells. PLoS ONE 2014, 9, e109868.

(c) 2018 by the authors. Licensee MDPI, Basel, Switzerland. This article is an open access article distributed under the terms and conditions of the Creative Commons Attribution (CC BY) license (http://creativecommons.org/licenses/by/4.0/). 